\title{
Remarques sur l'épidémiologie comparée des maladies parasitaires du lac d'Akosombo (Ghana) et du lac Nasser (Nubie Egypto-Soudanaise)
}

\author{
par R. DESCHIENS (*) \\ Laboratoire d'Epidémiologie de la Société de Pathologie exotique, Institut Pasteur, \\ 28, rue du Docteur-Roux, F 75015 Paris
}

\begin{abstract}
Résumé
L'étude écologique et épidémiologique comparée, des endémies parasitaires à vecteurs aquatiques, du lac d'Akosombo, au Ghana, et du lac Nasser, en Nubie Egypto-Soudanaise, fait ressortir deux aspects différents: le lac d'Akosombo, situé en région chaude et humide, est un système lacustre eutrophe où le paludisme, les bilharzioses et l'onchocercose sont fortement implantés et d'ailleurs bien contrôlés sanitairement. Le lac Nasser est situé en territoire aride ou désertique, la densité humaine y est très faible; le paludisme et l'onchocercose y sont seulement potentiels et efficacement surveillés, mais la bilharziose à Schistosoma haematobium y est présente chez $30 \%$ des pêcheurs.
\end{abstract}

\section{Summary}

A comparative ecological and epidemiological study of parasitic endemies with aquatic vectors in Akosombo lake (Ghana) and Nasser lake (Nubia, Egyptian Sudan) lets appear two different aspects of the problem: Akosombo lake, situated in a warm and

(*) En amical hommage au $\mathrm{P}^{r}$ Henri Galliard qui a participé, avec nous, sur le terrain, à la prospection du Lac d'Akosombo. 
damp country, is an eutrophic lacustral system where malaria, bilharziosis and onchocercosis are strongly implanted and besides well controlled. Nasser lake is situated in a arid and desertic soil where human density is very low; malaria and onchocercosis exists only potentially and are efficiently controlled; but Schistosomiasis haemabbium is present in $30 \%$ of the fishers.

L'implantation de barrages hydro-électriques ou d'irrigation à des fins industrielles et agricoles, dans les régions chaudes et tropicales, a, le plus souvent, pour corollaire la création de bassins lacustres (lacs de retenue, lacs d'équipement, «man made lakes» des auteurs anglo-saxons) qui peuvent représenter des volumes et des surfaces d'eau énormes entraînant des modifications géographiques, climatiques et écologiques considérables.

De tels bouleversements, qui sont parfois l'équivalent de cataclysmes naturels, peuvent avoir une incidence importante sur la santé publique particulièrement par la voie du développement des grandes endémies parasitaires (paludisme, bilharzioses, onchocercose) dont le complexe pathogène comporte des invertébrés vecteurs (Moustiques, Simulies, Mollusques) vivant, soit à l'état larvaire, soit à l'état adulte, dans l'eau, ou, encore, par celle du développement d'helminthes dont le cycle évolutif s'accomplit dans le sol humide (ankylostomose, nécatorose).

Il y a une pathologie des lacs d'équipement et des grands barrages tropicaux, qui pourrait d'ailleurs inclure certaines arboviroses.

En fonction de la situation géographique, géologique et climatique de ces grandes étendues lacustres artificielles, on peut être en présence de biotopes et de biocénoses se rassemblant en écosystèmes nouveaux appelés à modifier, à leur tour, l'aspect des problèmes sanitaires régionaux.

Un lac d'équipement établi dans une région désertique ou aride comme le lac Nasser (Haut-barrage d'Assouan sur le Nil) qui s'étend sur $7.000 \mathrm{~km}^{2}$ environ, qui est encadré par les déserts Lybique et Nubique, où il ne pleut pratiquement jamais et où la population humaine est très faible, ne pose pas, dans son périmètre et dans son hinterland, les mêmes problèmes écologiques, épidémiologiques et sanitaires qu'un lac d'équipement aménagé dans un environnement à pluies abondantes, à végétation luxuriante, et à population humaine relativement dense, comme le lac d'Akosombo, au Ghana, qui s'est développé, en corollaire du barrage de la Volta et qui occupe une surface de près de $9.000 \mathrm{~km}^{2}$.

L'étude comparée, sur les versants écologique et sanitaire (grandes endémies parasitaires) de ces deux systèmes lacustres d'équipement, fait ressortir les aspects suivants :

Le lac d'Akosombo est, actuellement (après cinq ans de mise en eau), un lac eutrophe dont la production en matière vivante ou «biomasse » (flore et faune aquatiques) est très élevée; les poissons y sont en grande abondance; la pêche et la fourniture d'énergie électrique représentent les deux grandes richesses économiques du lac. 
Du point de vue démographique, la densité de la population humaine (pêcheurs, cultivateurs et pasteurs, personnel employé au barrage), dans le périmètre du lac, est forte et atteint 100.000 personnes environ.

Sur le plan épidémiologique et sanitaire, les populations de vecteurs aquatiques (Moustiques, Mollusques, Simulies) du paludisme, des bilharzioses et des onchocercoses sont nombreuses et les trois endémies parasitaires humaines, sous revue, sévissent, bien que maintenues et contrôlées par des Services médicaux efficaces.

Les problèmes sanitaires liés à la pathologie des lacs d'équipement, dans les régions tropicales, sont ici posés pleinement pour le périmètre du lac et, pour partie dans son hinterland.

Bien entendu, les endémies rencontrées correspondent à une simple amplification régionale des complexes pathogènes qui frappaient le territoire antérieurement à la construction du barrage.

Le complexe du lac Nasser (Haut-barrage du Nil à Assouan) écologiquement différent du précédent, correspond à une immense cuvette allongée du Sud au Nord, entourée de déserts et pratiquement sans végétation rivulaire et terrestre et sans peuplements humains ou animaux importants, à l'exception de la ville d'Assouan située immédiatement en aval du barrage; c'est actuellement un lac oligotrophe dont la faune et la flore aquatiques sont relativement pauvres et dont la biomasse est, par conséquent, faible. Les herbiers de Chara ou de plantes immergées y sont rares; les plantes rivulaires très localisées. Les poissons sont en médiocre quantité; la pêche artisanale est, néanmoins, pratiquée par 3.000 Nubiens, environ, nomadisants et d'un contrôle difficile.

Les Invertébrés aquatiques, vecteurs potentiels du paludisme et de la bilharziose, sont présents, mais dispersés en petites niches écologiques.

Néanmoins, on ne doit pas oublier que lorsqu'il existait, dans l'ancienne Nubie, aujourd'hui submergée par les eaux du lac Nasser, des oasis, des villages et des habitants, le paludisme, la bilharziose à $S$. haematobium, l'onchocercose ainsi que leurs vecteurs, y étaient endémiques.

L'effacement, par immersion, des oasis, de leurs aménagements agricoles ainsi que la raréfaction de la population humaine ne semblent pas poser actuellement de problèmes sanitaires majeurs dans le périmètre du lac ; ces problèmes étant reportés sur les nouvelles surfaces d'irrigation créées à l'amont (Soudan) et à l'aval (HauteEgypte) du système lacustre.

Cependant, des foyers résiduels de bilharziose à S. haematobium (30\% des pêcheurs étaient contaminés en 1971) et des colonies de Bulinus truncatus se sont maintenues dans le périmètre du lac et cet ensemble représente une potentialité infectieuse préoccupante.

D'autre part, bien qu'il n'ait pas été signalé officiellement de cas de paludisme dans le périmètre du lac depuis 1956, l'un des vecteurs de cette parasitose Anopheles pharoensis peut être identifié sur son territoire bien qu'il y soit rare. En outre, il faut rappeler que, dans le passé, A. gambiae, vecteur du paludisme à Plasmodium 
falciparum, au Soudan, a fait - venant du sud - plusieurs incursions en Nubie en développant des endémo-épidémies sévères et que, dans le sud du lac, donc vers le Soudan, de petits systèmes d'eau rivulaire représentent d'excellentes niches écologiques pour $A$. gambiae.

Enfin, il convient de rappeler que l'onchocercose à Onchocerca volvulus et l'un de ses hôtes intermédiaires, Simulium damnosum, existaient en Nubie avant la création du Haut-barrage. Sans doute les gîtes à simulies ont-ils été noyés avec les végétations arbustives et les oasis nubiens, mais de nouveaux aménagements agricoles avec des eaux courantes pourraient les reconstituer dans l'avenir. On sait que l'onchocercose est endémique dans la région de Dongola, au Soudan, à $300 \mathrm{~km}$ en amont du lac Nasser et on connaît la capacité de dispersion des simulies, par le vent, lorsque ces insectes groupés prennent de l'altitude. Une menace potentielle d'onchocercose intéressant la Nubie n'est donc pas exclue d'autant plus qu'en aval du Haut-barrage, l'eau circule tourbillonnante et bordée de végétations rivulaires créant des conditions favorables à des gîtes de Simulies.

L'étude comparée, sur le versant écologique et épidémiologique, relativement aux grandes endémies parasitaires à vecteurs aquatiques, du lac d'Akosombo et du lac Nasser, fait ainsi ressortir deux aspects différents : le lac d'Akosombo est un système lacustre eutrophe en pleine production, situé dans une région chaude et humide, à végétation luxuriante et à densité démographique relativement forte ; dans cet environnement, les complexes pathogènes, en particulier ceux du paludisme, des bilharzioses et de l'onchocercose, sont fortement installés et heureusement bien contrôlés sanitairement.

Le lac Nasser correspond à un système lacustre encore oligotrophe à faible production, situé dans une région aride ou désertique, à peuplements végétaux et animaux réduits et à faible densité humaine (Assouan excepté). Les complexes pathogènes, singulièrement ceux concernant le paludisme, les bilharzioses et l'onchocercose, y sont ou très atténués, ou potentiels, mais constituent néanmoins une menace à venir d'autant plus accusée que les parasitoses sous examen sont endémiques dans la vallée du Nil, soit en amont, soit en aval du lac.

Les services sanitaires égyptiens du lac Nasser exercent d'ailleurs un contrôle épidémiologique et prophylactique des plus efficaces sur ces potentialités.

Sur les plans de la coordination des projets économiques (industrie, agriculture), techniques (géologie, art de l'ingénieur) et des préoccupations de santé publique (ép1démiologie, prophylaxie), relatifs à l'aménagement des grands barrages et de leurs lacs d'équipement dans les régions chaudes et tropicales, il apparaît qu'on ne peut évidemment choisir le point d'établissement du barrage d'un fleuve, qu'en fonction de possibilités géographiques, géologiques, techniques, économiques et démographiques. Les fins d'aménagement d'un barrage sont le progrès social et l'enrichissement des Etats. On construit où il faut et le mieux que l'on peut, mais ceux qui ont, sur le terrain, 
contribué au schéma de l'organisation technique, de la coordination, ainsi qu'à l'établissement du calendrier de la construction d'un grand barrage, savent combien sont lourdes et difficiles les tâches des ingénieurs et quelles sont leurs responsabilités: il faut les laisser travailler librement.

Le rôle du Service de Santé, par rapport à celui du maître d'œuvre, apparaît déjà, pendant la construction, pour assurer la sécurité médicale des ouvriers et des cadres sur le chantier; il interviendra ensuite dans toute la charge de sa responsabilité avec la surveillance et le quadrillage : nosologique, épidémiologique et prophylactique, du territoire aménagé.

Il apparaît, d'autre part, comme très utile, qu'au cours de l'élaboration et de la discussion du plan du barrage, l'autorité sanitaire soit représentée dans le Comité consultatif assistant le Directeur des Travaux, conjointement aux géologues, aux économistes, aux agronomes et aux écologistes. 